$$
Z^{p}-X^{p-1} Z-1=\prod_{i=0}^{p-1}\left(Z+i X-\sum_{i=0}^{\infty} X^{1-p^{-i}}\right)
$$

These factorizations can be verified directly. They were used in discovering some of the examples discussed in [1].

\title{
BIBLIOGRAPHY
}

1. S. Abhyankar, On the ramification of algebraic functions, Amer. J. Math. vol. 77 (1955) pp. 575-592.

2. C. Chevalley, On the theory of local rings, Ann. of Math. vol. 44 (1943), pp. 680-708.

3. - Introduction to the theory of algebraic functions of one variable, New York, 1951.

4. S. MacLane and O. F. G. Schilling, Zero-dimensional branches of rank one on algebraic varieties, Ann. of Math. vol. 40 (1939) pp. 507-520.

HARVARD UNIVERSITY

\section{ON THE COMPOSITUM OF ALGEBRAICALLY CLOSED SUBFIELDS}

\section{SHREERAM ABHYANKAR}

Professor Igusa asked me the following question: Given a field $K$, is the compositum of all the (absolutely) algebraically closed subfields of $K$ itself algebraically closed (of course, we are assuming that this compositum is not empty, i.e., that $K$ contains the algebraic closure of its prime field)? We shall show in $\$ 1$ that the answer to this question is in the negative in general. In \$2, we shall give a special case in which the answer is in the affirmative.

1. The algebraic closures of $k(x), k(y)$ and $k(x, y)$. Let $k$ be an arbitrary algebraically closed field, $L=k(x, y)$ where $x$ and $y$ are algebraically independent over $k, L^{*}=$ an algebraic closure of $L, M=k(x)$, $N=k(y), M^{*}=$ the algebraic closure of $M$ in $L^{*}, N^{*}=$ the algebraic closure of $N$ in $L^{*}$, and $K=$ the compositum of $L^{*}$ and $M^{*}$. Let $T$ be the compositum of all the algebraically closed subfields of $K$. Then $M^{*} C T$ and $N^{*} \subset T$ and hence $T=K$. We shall prove below that $K$ cannot be algebraically closed. In fact we shall show that in some sense $K$ is much nearer to $L$ than it is to $L^{*}$ and hence that $K$ is far from being algebraically closed.

Embed $L=k(x, y)$ canonically into the formal power series field

Received by the editors August 23, 1955. 
$L_{1}=k((x, y))$. Let $L_{1}^{*}$ be an algebraic closure of $L_{1}$. Then we may assume that $L^{*}$ is the algebraic closure of $L$ in $L_{1}^{*}$. Let $M_{1}=k((x)) \subset L_{1}$, $N_{1}=k((y)) \subset L_{1}$, and let $M_{1}^{*}$ and $N_{1}^{*}$ be the algebraic closures respectively of $M_{1}$ and $N_{1}$ in $L_{1}^{*}$. Let $K_{1}$ be the compositum of $M_{1}^{*}$ and $N_{1}^{*}$. We thus have: $k(x, y)=L \subset K \subset L^{*} \subset L_{1}^{*}$ and $K \subset K_{1}$. Let $p$ be the characteristic of $k$. To prove that $K$ is not algebraically closed (and that it is far from being algebraically closed) we shall show that if $F(Z)$ is an element of $K[Z]$ of the form

$$
F(Z)=Z^{n}-g(x)-h(y),
$$

where $g(x)$ is an arbitrary element of $k[x]$ with $g(0) \neq 0, h(y)$ is an arbitrary element of $k[y]$ with $h(0) \neq 0$, and $n$ is an integer greater than one with $n \neq 0(p)$ if $p \neq 0$ and otherwise arbitrary, then $F(Z)$ has no linear factor in $K[Z]$ (and hence in particular if $n$ is prime then $F(Z)$ is irreducible in $K[Z])$. We shall prove the stronger assertion that $F(Z)$ has no linear factor in $K_{1}[Z]$, and in proving this we shall let $g(x)$ be an arbitrary element of $k[[x]]$ with $g(0) \neq 0$ and $h(y)$ an arbitrary element of $k[[y]]$ with $h(0) \neq 0$ (i.e., $g(x)$ and $h(y)$ are power series respectively in $x$ and $y$ with coefficients in $k$ and of positive leading degrees). Assume the contrary, i.e., that $F(Z)$ has a linear factor $Z-q$ in $K_{1}[Z]$. Then $q^{n}=g(x)+h(y)$. Since $q$ is in the compositum $K_{1}$ of $M_{1}^{*}$ and $N_{1}^{*}$, we can write $q=\left(\sum_{i=1}^{m} a_{i} b_{i}\right) /\left(\sum_{i=1}^{m} c_{i} d_{i}\right)$ with $a_{i}, c_{i} \in M_{1}^{*}$ and $b_{i}, d_{i} \in N_{1}^{*}$ for $i=1,2, \cdots, m$, where $m$ is some positive integer. Therefore, there exist finite algebraic extensions $M_{2}$ and $N_{2}$ of $M_{1}$ and $N_{1}$ respectively such that $a_{i}, c_{i} \in M_{2}$ and $b_{i}, d_{i} \in N_{2}$ for $i=1,2, \cdots, m$. Let $r^{*}$ and $s^{*}$ be the leading degrees of $g(x)$ and $h(y)$ respectively. Let $\left[M_{2}: M_{1}\right]=r$ and $\left[N_{2}: N_{1}\right]=s$. By extending $M_{2}$ and $N_{2}$ suitably, we can arrange matters so that $r r^{*}=s s^{*}=t$ say. Then $M_{2}=k((u))$ and $N_{2}=k((v))$ with $x=u^{r} e(u)$ and $y=v^{8} f(v)$ where $e(u)$ and $f(v)$ are units in $k[[u]]$ and $k[[v]]$ respectively. Let $H$ be the compositum of $M_{2}$ and $N_{2}$. Let $g^{*}(u)$ and $h^{*}(v)$ be the elements of $k[[u]]$ and $k[[v]]$ gotten by substituting $u^{r} e(u)$ and $v^{8} f(v)$ for $x$ and $y$ in $g(x)$ and $h(y)$ respectively. Then the polynomial

$$
F^{*}(Z)=\left[Z^{n}-d^{*}(u, v)\right] \in H[Z]
$$

where $d^{*}(u, v)=g^{*}(u)+h^{*}(v) \in k[[u, v]]$, has a linear factor in $H[Z]$. We shall prove that this is a contradiction by proving more strongly that $F^{*}(Z)$ has no linear factor in $L_{2}[Z]$ where $L_{2}=k((u, v))$. Let $d(u, v)$ be the leading form of $d^{*}(u, v)$. Now, the leading degree of $g^{*}(u)$ in $u=r r^{*}=t=s s^{*}=$ the leading degree of $h^{*}(v)$ in $v$. Therefore, $d(x, y)$ $=a u^{t}+b v^{t}$ where $a$ and $b$ are nonzero elements of $k$. Suppose, if possible, that $F^{*}(Z)$ has a linear factor $Z-w^{*}(u, v)$ with $w^{*}(u, v) \in L_{2}$. 
Since $k[[u, v]]$ is integrally closed in $L_{2}$, we must have $w^{*}(u, v)$ $\in k[[u, v]]$. Let $w(u, v)$ be the leading form of $w^{*}(u, v)$. Then $w^{*}(u, v)^{n}$ $=d^{*}(u, v)$ and hence $w(u, v)^{n}=a u^{t}+b v^{t}$; this is a contradiction (since $n \neq 0(p)$ if $p \neq 0)$.

2. Finitely generated extensions. Let now $k$ be an algebraically closed field and let $K$ be a finitely generated field extension of $k$. Let $T$ be the compositum of all the algebraically closed subfields of $K$. Then we have that $T=k$ or equivalently we may state the

Proposition. Let $L$ be an algebraically closed subfield of $K$. Then $L \subset k$.

Proof. Otherwise we can find an element $x$ in $L$ which is transcendental over $k$. Let $P$ be the prime field of $k$ and let $P^{*}$ be the algebraic closure of $P$ (in $K$ ). Then $P^{*} \subset k \cap L$. Let $Q=P^{*}(x)$ and $Q^{*}=$ the algebraic closure of $Q$ (in $K$ ). Then $Q^{*} C L$. Let $M$ be the compositum of $k$ and $Q^{*}$. Then $M$ is finitely generated over $k$ and hence $[M: k(x)]$ $=n<\infty$. Now we may either use elementary properties of polynomials or alternatively we may use valuations.

To invoke valuations, let $v$ be the valuation of $k(x) / k$ for which $v(x)=1$, where $v$ is normalized so as to have the additive group of integers as its value group. Let $w$ be an extension of $v$ to $M$. Then for any nonzero element $a$ of $M$ with $w(a)>0$ we must have: $w(a) \geqq 1 / n$. Now $Q^{*} \subset M$ implies that for any integer $m$ there exists $y_{m}$ in $M$ with $y_{m}^{m}=x$. Then $w\left(y_{m}\right)=(1 / m) \quad v(x)=1 / m$ and hence $0<w\left(y_{m}\right)<1 / n$ whenever $m>n$; this is a contradiction. Therefore $L \subset k$.

Alternatively, to use an elementary argument, let $m$ be an integer greater than $n$. Then there exists an element $y_{m}$ in $Q^{*} \subset M$ such that $y_{m}^{m}=x$. Now, the polynomial $X^{m}-x$ is irreducible in $k[X, x]$ ( $X$ an indeterminate). Therefore $X^{m}-x$ is also irreducible in $k(x)[X]$. Hence $n=[M: k(x)] \geqq\left[k(x)\left(y_{m}\right): k(x)\right]=m$, which is a contradiction. Therefore $L \subset k$.

We observe that the method of $\S 2$ (of valuations) can be applied to prove the assertions of $\S 1$ and conversely the method of $\$ 1$ (of power series) can be applied to prove the proposition of $\$ 2$.

HARVARD UNIVERSITY 